\title{
Adversaire honorable ou barbare vicieux ? La perception de l'ennemi sous la Révolution et l'Empire
}

Honourable opponent or savage barbarian: the perception of the enemy during the Revolution and Empire

\section{Alan Forrest}

\section{(2) OpenEdition} Journals

\section{Édition électronique}

URL : https://journals.openedition.org/ahrf/12588

DOI : $10.4000 /$ ahrf. 12588

ISSN : 1952-403X

\section{Éditeur :}

Armand Colin, Société des études robespierristes

Édition imprimée

Date de publication : 1 septembre 2012

Pagination : $5-24$

ISBN : 978-2-200-92761-5

ISSN : 0003-4436

\section{Référence électronique}

Alan Forrest, « Adversaire honorable ou barbare vicieux ? La perception de l'ennemi sous la Révolution et l'Empire ", Annales historiques de la Révolution française [En ligne], 369 | juillet-septembre 2012, mis en ligne le 01 septembre 2015, consulté le 24 avril 2022. URL : http://journals.openedition.org/ahrf/ 12588 ; DOI : https://doi.org/10.4000/ahrf.12588 


\title{
ADVERSAIRE HONORABLE OU BARBARE VICIEUX? LA PERCEPTION DE L'ENNEMI SOUS LA RÉVOLUTION ET L'EMPIRE
}

\author{
Alan FORREST
}

\begin{abstract}
Dans le langage politique de la Révolution, les ennemis de la France sont souvent dépeints dans les termes les plus atroces, comme des terroristes barbares ou les laquais de lâches tyrans, l'identité française se construisant par opposition à celle de ses adversaires dans la guerre. Avec l'élargissement impérial du conflit, avec la création d'une armée multinationale et multilingue, cette image assez stéréotypée s'est cependant complexifiée. L'expérience de la guerre a suscité des réponses variées face à l'ennemi, qui ont pu inclure le respect et même l'amitié ; et les écrits des soldats pendant les guerres napoléoniennes suggèrent que certaines perceptions sont encore inspirées de l'idéologie révolutionnaire. Ces écrits contiennent ainsi un éventail d'opinions qui rappellent les récits de voyage du XVIII siècle, affichent un goût pour l'exotisme et trahissent les anciens préjugés concernant la supériorité de la civilisation européenne occidentale.
\end{abstract}

Mots-clés : voyage, ego-documents, exotisme, soldat, adversaire, barbare

Le 16 juin 1809, deux jours après la bataille de Raab en Autriche, le cavalier français Jacques Chevillet décrit dans sa correspondance un des soldats de l'ennemi avec qui il avait dû combattre. C'est un homme comme lui, qui partage les mêmes réflexes, les mêmes craintes, les mêmes menus plaisirs ; il ne le dépeint pas en monstre. Mais ce qui le frappe en ce moment d'hostilité, c'est surtout la différence : la corpulence, la menace dans ses yeux, les divers comportements qui le distinguent du militaire français. Chevillet décrit ainsi le moment où il le somme de se rendre : 
« ce soldat s'arrêta tout à coup, me fit face en me croisant sa baïonnette ; il me répondit dans un langage croate que je ne compris pas ». Monté à cheval, le jeune Français tourne autour de lui, « ce qui [lui] donna le temps de considérer ce vilain soldat qui voulait me braver ». Et que perçoit-il devant lui ? Un homme à la fois grossier et vulnérable, brutal et peureux.

"C'était un gros balourd croate tout brute, ressemblant plutôt à un sauvage qu'à un soldat ordinaire. Sa veste toute déboutonnée laissait voir sa large poitrine à découvert; il avait la figure et le col tout noircis de crasse et de poudre, il avait perdu son chapeau, car il avait la tête nue avec une grosse chevelure noire touffue et hérissée dont une partie lui couvrait les yeux ».

Dans les circonstances de cette réunion malchanceuse c'est surtout un ennemi, qu'il faut tuer pour ne pas risquer d'être tué son tour. Le temps manque pour ces petits gestes qui auraient pu établir entre eux-mêmes une trace de fraternité. Comme ennemi il ne peut qu'être assommé, sinon exterminé. Chevillet conclut :

« Je lui récidivai avec menace de jeter son fusil. Alors soit crainte, soit envie de se sauver, il marchait à reculons sans néanmoins cesser de me croiser sa baïonnette. Son opiniâtreté ne servait qu'à m'actionner davantage ; en deux ou trois sauts de mon cheval, j'arrivai sur sa gauche et l'assommai d'un furieux coup de sabre sur la tête, dont je le vis tomber sur-le-champ $»^{1}$.

En temps de guerre, la mort d'un adversaire est un événement quotidien, et le fait de tuer un autre être humain n'évoque aucune émotion exceptionnelle. C'est la réponse qui est attendue de lui, et il n'y a aucune raison de supposer que cette tuerie le troublerait ou interromprait son sommeil. Lui-même fils de soldat, né dans la société très militarisée de La Fère en Picardie, Chevillet avait passé sa jeunesse dans l'ombre de l'armée, qu'il rêvait d'intégrer ${ }^{2}$. Mais sa description du Croate est exceptionnelle par le détail qu'elle offre, et on peut y décerner un certain intérêt, une sympathie même, pour cet homme qu'il vient de massacrer et qu'il compare à un « sauvage », intérêt né d'une expérience personnelle de la guerre et d'une nouvelle familiarité avec les habitants d'autres pays européens, même des

(1) Jacques CHEVILLET, Souvenirs d'un cavalier de la Grande Armée, 1800-1810, préface de Henry Houssaye, annoté par Christophe Bourachot Paris, 2004, p. 229.

(2) Ibid., Présentation de Christophe Bourachot, p. 7. 
plus lointains. Avant de passer en Autriche, le jeune trompette, qui s'était engagé à l'âge de quinze ans, avait déjà fait un voyage remarquable au service de l'Empereur, passant de la Hollande en Allemagne, de l'Italie en Autriche et en Hongrie. Ce voyage, qu'il vit presque comme un rite de passage de l'adolescence à l'âge adulte, l'expose à tout le continent européen. De plus en plus, les hommes contre qui il s'engage ne sont plus des inconnus ou des barbares à craindre ; ce sont des Européens comme lui, dont il avait rencontré les proches dans les villages qu'il avait parcourus pendant sa longue marche. Des Européens, d'ailleurs, avec qui il est possible de s'entretenir, se servant de ce qu'ils ont de langue commune.

Le même Chevillet raconte qu'en 1809, en Italie, près du village de Baglione, lui et un camarade avaient croisé deux hussards de l'armée autrichienne, qui, loin de s'attaquer à eux, leur avaient fait signe de s'approcher et les avaient invités à boire un coup. Chevillet a du vin du pays dans sa gourde, tandis que l'Autrichien avec qui il s'entretient tient dans sa main une bonne eau-de-vie. Au début, bien sûr, les Français restent soupçonneux, craignant un piège. Mais la curiosité et la sociabilité du soldat l'emportent, et au bout de quelques moments de tension, parlant italien et sous le regard de leurs compagnons, les deux hommes, dans une scène qui évoque la Grande Guerre, se permettent de fraterniser, chacun traversant un bras de la rivière pour gagner une petite île, terre démilitarisée, où ils passent un bon quart d'heure ensemble, buvant à la santé de l'autre. Chevillet explique dans une lettre à son père ce qui est pour lui la signification d'un moment qu'il prise beaucoup. " Alors nous bûmes chacun dans nos gourdes à la santé l'un de l'autre et nous nous offrîmes à boire réciproquement, moi du vin, et lui de l'eau-de-vie, à trois ou quatre reprises $[\ldots] »$.

"C'est ainsi, mon père », ajoute-t-il, « qu'un trompette du $8^{\mathrm{e}}$ régiment de chasseurs à cheval, votre fils, et un hussard du régiment Marie-Thérèse, qui se font une guerre à mort, nous semblions nous réconcilier et faire la paix en buvant ensemble avec une égale confiance. De la manière dont nous nous accueillîmes, l'on eût dit que nous nous connaissions et que nous étions amis plutôt qu'ennemis ; le hussard avait été en France et il conservait de l'estime pour les Français $»^{3}$.

Cet incident montre à quel point la vie de militaire peut, lorsque les circonstances y sont favorables, contribuer à dissoudre les animosités entre 
hommes et cultures, même entre adversaires. Leur volonté de se connaître résulte de leur déplacement à travers l'Europe et d'une première rencontre avec d'autres peuples et cultures, qui élargit les horizons et qui marque le soldat pour la vie. Elle affecte autant le conscrit arraché de mauvais gré à son village natal que l'officier de carrière dont la vie est caractérisée par une mobilité quasi-permanente. Aucune génération n'avait si loin voyagé que celle des années 1790 et 1800, années dominées par les guerres en Europe et au-delà. Aucune autre ne le ferait, d'ailleurs, au moins à cette échelle, avant la guerre de 1914-1918.

\section{Le soldat voyageur}

Certes, le soldat a toujours été voyageur, et le goût de l'aventure et de l'exotique figure parmi les plaisirs séculaires de la guerre. Le voyage compte parmi les raisons les plus citées par les individus d'origine aisée, qui optent pour la carrière des armes. Les voyages sont racontés d'une façon quasi-routinière dans les lettres familiales comme sur les pages des carnets et des mémoires, voyages caractérisés par la découverte et par l'aventure. Quelques-uns, comme dans toutes les générations, cherchent dans le service militaire l'occasion de s'échapper, de quitter leur famille et leur village pour la première et peut-être la seule fois de leur vie, à la découverte de l'inconnu. Cela fait de ces années de guerres révolutionnaires et napoléoniennes, soit en Europe, soit en Afrique du Nord ou dans les colonies de la Caraïbe, une ère qui privilégie le voyage et les migrations temporaires. En France, des jeunes gens qui ne se seraient jamais aventurés plus loin que la ville de marché la plus proche rencontrent des gens d'ailleurs et entendent des langues et des patois inconnus ; et, une fois au-delà des frontières, ils font la connaissance de tout un continent, ses paysages et ses grandes villes, ses châteaux et ses cathédrales, ses cultures et ses peuples. C'est grâce à la guerre qu'ils deviennent des voyageurs intrépides, grands connaisseurs de l'Europe, suivant les pas de ces femmes et ces hommes qui avaient, sous l'Ancien Régime, profité de leur situation aisée pour faire le Grand Tour et s'adonner aux plaisirs du voyage. Parmi les officiers des bataillons révolutionnaires, comme de la Grande Armée de l'Empereur, on trouve bien des individus qui eux-mêmes font cette comparaison, et qui, en lecteurs avides, empruntent à la littérature du voyage, aux auteurs voyageurs les plus connus, le style et la curiosité. Ce qui leur fournit, bien sûr, même avant le départ, des attentes, des présuppositions, une vision de ce qu'ils vont trouver dans les pays qu'ils traversent. Le soldat voyageur, tout comme son homologue civil, est influencé par les écrits des autres, 
de ceux, militaires et civils, qui l'ont précédé. Son récit est coloré par les réflexions qu'il a assimilées, souvent longtemps avant son départ, et qui sont déjà formulées dans son imagination.

Le XVIII ${ }^{\mathrm{e}}$ est une période exceptionnelle pour le voyage, autant en France qu'ailleurs en Europe. Le Grand Tour avait été l'invention du Nord, des pays froids, commerciaux et protestants qui manquaient d'une culture latine, et qui étaient pressés par l'envie de se rapprocher de plus près de leurs racines classiques. Le romancier Anthony Burgess capte bien cet esprit. « Si le Germain ou l'Anglais ne souhaite pas s'abandonner, si peu que ce soit, à l'influence du Sud », écrit-il avec peut-être un brin d'exagération, « le danger demeure toujours qu'il retombe dans la grossièreté au mieux, la brutalité au pire $»^{4}$. Les familles aristocratiques donnent le ton à une tradition de voyage et d'instruction qui privilégie surtout l'Italie, prisée par des générations élevées dans le respect de toute la civilisation de Rome et de Grèce. Mais, puisque la Grèce est trop lointaine, c'est donc en Italie qu'ils affluent, attirés par la grandeur de l'architecture des villes et par les ruines d'une civilisation disparue. Mais s'ils sont prêts à admirer ces ruines, le fait même que celles-ci ont été laissées à l'abandon leur suggère une société en pleine décadence, un pays déclinant, à l'écart de la civilisation moderne ${ }^{5}$. Les ruines de l'Antiquité sont, depuis la Renaissance, une source d'inspiration pour ceux qui s'identifient à une civilisation judéo-chrétienne, d'où l'attrait quasi-permanent des pays méditerranéens. D'autres villes et d'autres pays ont leurs touristes, bien sûr, notamment la France des Lumières, la Suisse et Genève, qui est perçue comme la ville de JeanJacques Rousseau et comme un des berceaux de la philosophie moderne. Mais l'important, c'est qu'on voyage pour regarder, pour s'instruire, pour assimiler quelques vestiges de l'esprit des peuples et de l'atmosphère des lieux. Bien sûr, l'image du Grand Tour reste liée au plaisir et à la frivolité : l'art et la littérature touristiques de l'époque sont dominés par des représentations statiques des panoramas et des curiosités visités ${ }^{6}$. Mais au cours du XVIII ${ }^{\mathrm{e}}$, le tourisme devient plus sérieux, plus sophistiqué. Tout voyageur veut passer pour artiste et pour ethnologue ; tous, même les plus

(4) Anthony Burgess, « Le voyage en Europe », dans Anthony BURGESS et Francis HaSKELL (dir.) Le Grand Siècle du voyage, Paris, 1968, p. 13.

(5) Jeremy BLACK, Italy and the Grand Tour, New Haven, Connecticut, 2003, p. 7.

(6) Chloe CHARD, «Introduction », dans Chloe CHARD et Helen LANGDON (dir.), Transports: Travel, Pleasure and Imaginative Geography, 1600-1830, New Haven, Yale University Press, 1996, p. 25. 
aristocrates, subissent l'influence d'une nouvelle génération de touristes issue des mondes du commerce et de la science ${ }^{7}$.

Les voyageurs français sont parmi les pionniers, même s'ils ne partagent pas pleinement le goût du voyage qu'on décèle outre-Manche. Tout comme les Anglais, ils montrent une préférence pour l'Italie, avec ses antiquités et ses richesses artistiques. Ceux qui y passent après le milieu du siècle profitent du grand nombre des ouvrages qui leur ouvrent les portes des grandes villes et leur expliquent la beauté des sites archéologiques. En 1758, on publie le Voyage d'Italie de Cochin, qui déclenche, nous dit Gilles Bertrand, une tradition de "guides volumineux où dominent les récits de Richard et de Lalande, marqués par l'esprit encyclopédique »; il est à remarquer que les gens qui s'en servent sont généralement avides de culture, et que les guides contiennent un tas de renseignements détaillés, sur l'architecture et les monuments de l'Antiquité, bien entendu, mais aussi sur les sciences, la culture et la vie artistique. Le voyage est long, coûte cher et suit généralement un parcours fixe, prédéterminé, reprenant les pas des autres. Quant aux voyageurs eux-mêmes, ils évoluent lentement. Dans les années 1760 et 1770, le voyageur traditionnel, artiste, homme de lettres ou architecte, est concurrencé par une nouvelle clientèle du monde du négoce, ainsi que par les « hommes de science », parmi lesquels Lalande et Cassini. Sous la Révolution et l'Empire, il y a encore une évolution : la tradition du voyage est reprise par des écrivains, bien sûr, tels que Chateaubriand et Mme de Staël, mais aussi par des artistes et hommes de science à la solde du gouvernement, ainsi que par ceux qui nous intéressent le plus ici, «la vaste cohorte des militaires $»^{8}$. Leur regard sur les terres et les peuples qu'ils croisent dans leurs voyages est celui d'une génération de transition entre les Lumières et le Romantisme. Bien sûr ces voyages sont toujours encadrés par l'esprit de domination qu'impose l'Empire. Comme l'exprime avec une grande précision Nicolas Bourguinat, «l'on n'est pas toujours loin d'un regard ethnographique de type impérialiste, réordonnant la diversité de l'Europe conquise par rapport à la figure obligée du progrès qu'incarne la civilisation française $»$.

(7) Gilles BerTRAnd, Le Grand Tour revisité. Pour une archéologie du tourisme : le voyage des Français en Italie, milieu $18^{e}$-début $19^{e}$ siècle, Rome, Publications de l'École française de Rome, 2008, p. 489.

(8) Ibid., p. 10-11.

(9) Nicolas BOURGUINAT, « Un temps de rupture dans l'histoire des pratiques du voyage », dans Nicolas BOURGUINAT et Sylvain VENAYRE (dir.), Voyager en Europe de Humboldt à Stendhal : contraintes nationales et tentations cosmopolites, 1790-1840, Paris, Nouveau Monde éditions, 2007 , p. 15. 
Les écrits des gens de lettres sont sans doute les plus influents, avec un plus grand tirage et une meilleure distribution; et le style plus romantique de la fin du siècle et de la période des guerres napoléoniennes ne fait qu'accroître leur popularité et leur allure. Tout comme Walter Scott du côté des Anglais, Chateaubriand et Germaine de Staël sont lus et appréciés par le grand public, parmi lesquels des militaires, officiers de la Grande Armée. Et pour cause. Ce sont des auteurs bien accessibles, rendant d'ailleurs très bien l'atmosphère dans leur description des villes ou des mœurs des pays voisins où passeront à leur tour les militaires. Prenons par exemple De l'Allemagne de Mme de Staël, dans lequel elle donne ses opinions sur les villes allemandes qu'elle avait visitées, et dont elle décrit avec une perspicacité parfois acerbe les coutumes et les mœurs. Sur le charme des femmes en Allemagne, par exemple, elle écrit qu' «elles cherchent à plaire par la sensibilité, à intéresser par l'imagination ; la langue de la poésie et des beaux-arts leur est connue ; elles font de la coquetterie avec de l'enthousiasme, comme on en fait en France avec de l'esprit et de la plaisanterie $»^{10}$. Quel officier de la Garde pourrait ne pas être séduit par ce portrait des femmes allemandes, davantage que par cette description limpide de la politesse étouffante de la haute société viennoise ?

«L'exactitude de la politesse, qui est à quelques égards une vertu, a introduit dans Vienne les plus ennuyeux usages possibles. Toute la bonne compagnie se transporte en masse d'un salon à l'autre trois ou quatre fois par semaine. On perd un certain temps pour la toilette nécessaire dans ces grandes réunions ; on en perd dans la rue, on en perd sur les escaliers, en attendant que le tour de sa voiture arrive ; on en perd en restant trois heures à table, et il est impossible, dans ces assemblées nombreuses, de rien entendre qui sorte du cercle des phrases convenues ».

Pour conclure, Mme de Staël offre ce jugement fulgurant.

"C'est une habile invention de la médiocrité pour annuler les facultés de l'esprit, que cette exhibition journalière de tous les individus les uns aux autres. S'il était reconnu qu'il faut considérer la pensée comme une maladie contre laquelle un régime régulier est nécessaire, on ne saurait rien imaginer de mieux qu'un genre de distraction à la fois étourdissant et insipide : une telle distraction ne permet de suivre aucune idée, et transforme le

(10) Germaine de STAËL, De l'Allemagne (1810/1813), cité dans Anthony BURGESS et Francis HASKELL, Le Grand Siècle, op. cit., p. 83. 
langage en un gazouillement qui peut être appris aux hommes comme à des oiseaux $\gg^{11}$.

De l'Allemagne est un livre à savourer, difficilement réductible à un simple guide de voyage !

\section{Le goût de l'exotique}

Le XVIII produit également le goût de l'exotique, par des cultures et des pays orientaux, du voyage en dehors du vieux continent. Parmi les auteurs les plus appréciés à cet égard est Constantin-François, comte de Volney, connu pour une série d'ouvrages, notamment pour Les ruines, ou Méditations sur la révolution des empires, paru en 1791, et son Voyage en Syrie et en Egypte, publié quatre ans plus tôt suivant le voyage de l'auteur au Levant ${ }^{12}$. Le livre impressionne par sa méthode et ses détails. Il donne des renseignements sur les divisions ethniques en Égypte, sur les pratiques religieuses, sur les lois et surtout la loi sur la propriété, sur l'agriculture, l'histoire et les monuments historiques. Le texte inclut huit gravures, cartes et plans, y compris des représentations du Sphinx et des Pyramides de Gizeh qui ont suscité un intérêt particulier ; il comprend aussi des gravures sur quelques-uns des grands sites archéologiques de la région. Le livre a connu un grand succès. Il est paru en plusieurs éditions, dont la troisième, la plus complète, est publiée en 1799 , au moment même où l'expédition de Napoléon en Égypte suscite un nouvel intérêt dans le grand public. Ses lecteurs comprennent, bien sûr, des officiers et des scientifiques sur le point d'embarquer pour l'Afrique du Nord. Napoléon lui-même l'avait lu, avant son départ pour le Levant, et il ne cacha pas son admiration pour Volney et son Voyage, en déclarant dans le Moniteur que c'était « à peu près le seul livre qui n'eût pas menti $\gg^{13}$ - évidence, s'il en faut, qu'il y a dans cette fin de siècle turbulente un niveau d'intérêt extraordinaire pour les civilisations étrangères et un transfert culturel entre le littéraire et le militaire.

S'il est vrai que Napoléon peut être perçu comme exceptionnel en raison de sa passion pour la lecture et de sa formation éclairée - peu de généraux avaient lu les grands ouvrages classiques sur l'histoire et la guerre, ou partaient en campagne avec toute une bibliothèque en renfort

(11) Ibid., p. 89.

(12) Constantin-François Volney, Voyage en Syrie et en Egypte, Paris, 1787.

(13) Réimpression de l'Ancien Moniteur, 5 brumaire VIII ; Anne DENEYS-TUNNEY et Henri DENEYS, introduction à Volney, Euvres, t. III : Voyage en Syrie et en Egypte et Considérations sur la guerre des Turcs, Paris, Fayard, 1998, p. 5. 
- la culture de l'officier était celle d'un homme lettré, civilisé, avec une certaine ouverture sur le monde.

Les officiers français entrent dans les autres pays d'Europe avec dans la tête certaines attentes et présupposés qui résultaient de leurs lectures. Ils s'intéressent aux travaux des champs et à l'architecture des villes, même dans les pires circonstances; ils commentent les paysages, surtout les falaises, les défilés, les pics des montagnes, ces hauts lieux des touristes du Grand Tour, ces lieux qui inspirent un peu la crainte, voire le sens du danger. C'est une habitude qu'ils empruntent aux auteurs du XVIII ${ }^{\mathrm{e}}$, surtout ceux qui frôlent le romantisme. Ces paysages dramatiques se présentent comme des terres sauvages, éloignées, difficiles d'accès, un monde secret auquel le touriste s'abandonne. «L'île de Caprée, qui peut avoir environ dix milles de circuit, est partout environnée des plus hauts rochers; on n'y aborde, ainsi que je viens de vous le dire, que par le petit port qui est en face du golfe de Naples $\gg^{14}$. Cette phrase, du marquis de Sade, est typique de la littérature touristique de la période, et on la retrouve sous maintes formes dans les écrits de militaires, qui soulignent les aspects les plus dramatiques, les plus exotiques, les plus merveilleux des paysages qu'ils traversent. En le faisant ils satisfont les lecteurs de cette littérature du voyage qui les a accoutumés à lire des descriptions de l'exceptionnel, des vues rares, sublimes, écartées de l'expérience quotidienne. Les monuments historiques surtout sont présentés comme nus, solitaires, baignés de clair de lune. Comme ce Colisée perçu par Louis Simond dans son Voyage en Italie et en Sicile (1828) :

« La lumière douce et vague qu'il répandait sur les masses caverneuses entassées autour de l'arène, ne laissait voir aucun des tristes détails de la décadence, ni rien qui rappelât la règle et le compas. Une sorte de grandeur idéale, sans couleur et presque sans forme, se montrait seule, et, au lieu d'un ouvrage artificiel composé de murs et de voûtes, on aurait cru être au fond du cratère d'un volcan éteint dont le cône escarpé s'élevait à l'entour $»^{15}$.

Dans les mémoires militaires on trouve la même recherche de l'austère et du sublime.

(14) Chloe CHARD, Pleasure and Guilt on the Grand Tour: Travel writing and imaginative geography, 1600-1830, Manchester, Manchester University Press, 1999, p. 10.

(15) Louis SimOND, Voyage en Italie et en Sicile, Paris, 1828, vol. 1, p. 218, cité dans Chloe CHARD, Pleasure and Guilt, op. cit, p. 228. 


\section{Regards de militaires}

Mais ils regardent le pays aussi en militaires, toujours conscients des effets du paysage sur leur progression et des dangers qu'il pouvait cacher. Hippolyte d'Espinchal, par exemple, décrit la route qu'il doit suivre dans sa marche avec l'œil expérimenté du soldat. " La route était fort belle, mais dangereuse », écrit-il ; «nous marchions continuellement entre deux montagnes avec une petite rivière à notre gauche ». Ce passage, ajoute-t-il, est « fort renommé par les nombreuses attaques de guérillas » dont il est témoin. Et un peu plus loin, en s'approchant de Vittoria, il exprime des inquiétudes semblables.

« La route pour arriver à Vittoria est fort belle, mais resserrée jusqu'à Salinas, où se trouve une montagne rapide, dont la descente du côté de l'Espagne est douce et facile ; une plaine assez étendue se développe, mais des bois épais, avoisinant la route, offraient à l'ennemi la faculté de pouvoir dresser des embuscades et d'attaquer avec avantage $»^{16}$.

Même en ville, ses intérêts touristiques ne peuvent pas jouir d'une autonomie totale ; sa vision reste largement militaire, affectée moins par les paysages eux-mêmes que par leur potentiel stratégique. Il ne peut jamais voyager en touriste banal, sans référence aux potentialités pour l'armée ${ }^{17}$. À Ségovie, par exemple, il note combien la ville est belle, placée sur une hauteur au-dessus d'une rivière ; et il continue avec tout l'enthousiasme d'un touriste qui n'a pas négligé de lire un peu d'histoire locale :

«Les fortifications dont elle était entourée et une citadelle en bon état, assuraient la tranquillité de cette ville, autrefois fameuse à tant de titres et digne encore de l'attention des voyageurs par sa cathédrale et son Alcazar, bâti jadis et habité par les rois Goths, et par un aqueduc assez bien conservé, ouvrage de ce grand peuple qui a laissé partout des monuments de sa magnificence et de son immortalité. Une partie de l'Europe tire de Ségovie les meilleures laines que produise l'Espagne; il y a aussi une fort belle manufacture de glaces et plusieurs établissements d'un grand intérêt $»^{18}$.

(16) Michel Molières (dir.), Souvenirs militaires d'Hippolyte d'Espinchal, Paris, Le livre chez vous, 2005, p. 266-67.

(17) Natalie Petiteau, « Les voyages des hommes de la Grande Armée : de la vie militaire aux pratiques de la mobilité géographique », dans Nicolas BOURGUINAT et Sylvain VENAYRE, Voyager en Europe de Humboldt à Stendhal, op. cit., p. 363.

(18) Ibid., p. 277. 
Le soldat-touriste prend donc le temps de regarder et de s'informer. Le dragon Auguste Thirion, dans ses Souvenirs militaires, va plus loin ; il cherche à associer la culture et la géographie de l'Espagne avec le caractère de la guerre qu'il y rencontre, et ne cache pas son dédain pour un peuple qu'il juge mal intégré dans les mœurs européennes. Tout remonte au caractère du peuple et à ses vices, et au fait qu'il « reste étranger aux nations qui l'entourent »; au point qu'il peut affirmer que

« si l'Espagne avait été peuplée d'Allemands, de Polonais ou d'Italiens, après deux ou trois batailles gagnées, c'eût été un pays conquis dès que la capitale aurait été occupée ; mais on trouva un peuple ignorant, fanatique, sobre au milieu de l'abondance, tirant de sa sobriété, de sa manière de vivre, autant de vanité que les autres en tirent de leurs richesses, de leurs jouissances ».

À ses yeux l'Espagnol est suffisamment différent de l'Européen pour ne plus le compter comme tel. «C'est une erreur de la géographie que d'avoir attribué l'Espagne à l'Europe ; elle est africaine par le sang, les mœurs, le langage, la manière de vivre et de combattre ». Il l'explique avec un discours sur l'histoire de l'Espagne qui témoigne encore de ses lectures :

«Les deux nations ont été trop longtemps mêlées, les Carthaginois venus d'Afrique, les Vandales passés en Afrique et revenus d'Espagne, les Maures y séjournant pendant 700 ans, pour qu'une aussi longue cohabitation, pour que ces transfusions de peuples et de coutumes n'aient pas confondu ensemble les races et leurs mœurs $»^{19}$.

Dans son analyse on trouve des traces de Rousseau sur l'impact des climats chauds sur les peuples, et déjà l'accent mis sur ces transfusions sanguines qui préoccuperont Gobineau et les adeptes d'un darwinisme social. Pour Thirion, l'Espagnol passe déjà pour un étranger, un homme à part et autre, un homme avec tous les attributs de l'ennemi potentiel.

Car les réflexions de Thirion s'inspirent aussi de considérations militaires, et il offre ses observations sur l'implication de ces notations ethniques pour la conduite du combat. C'est, à son avis, l'explication qu'il faut chercher pour expliquer la guérilla et la manière inaccoutumée avec laquelle l'Espagnol fait la guerre. L'Espagnol, pour lui, 
« rappelle l'Arabe dévoré par le soleil, exerçant à la fois le brigandage ensanglanté et l'hospitalité, et réunissant en lui les extrêmes de la barbarie et de l'humanité ; enfin ce qui complète la ressemblance entre eux, c'est la similitude de leur manière de combattre. L'Occident attache l'honneur des armes à la précision des manœuvres, à l'immobilité des lignes, à l'opposition d'un front imperturbable à l'ennemi. Le soldat de l'Occident ne doit savoir marcher qu'en avant, et en arrivant sous le drapeau il s'est interdit de reculer. Le soldat de l'Orient, de l'Afrique, de l'Espagne s'est de tout temps affranchi de cette régularité ; pour lui, il n'y a pas de lignes ; pour lui, voltiger c'est combattre ; détruire l'ennemi, quel que soit le moyen, c'est le vaincre ; fuir, c'est l'attirer ou s'en préserver ; la victoire n'est pas comme en Occident à jour fixe et sur terrain donné, qu'importe qu'on ait abandonné un champ de bataille, un poste, une ville, si l'on peut y retourner le lendemain. Les idées de gloire et d'honneur qui sont l'âme des guerriers de l'Occident, sont dans l'Orient et le Midi remplacées par celle de la destruction de l'ennemi, comme seul objet de la guerre : que le trait frappe, on ne demande pas s'il a été lancé en fuyant. L'Espagnol est le Parthe de l'Europe $»^{20}$.

Ce qu'exprime avec éloquence Thirion - et cette perception est partagée par d'autres - est à la fois une explication de la guérilla et une vision comparatiste de deux sociétés de souches radicalement différentes.

\section{Le discours révolutionnaire}

Ailleurs on trouve une autre conception de l'ennemi, celle du discours politique de la Révolution qui avait pris racine et contribué puissamment à la perception de l'autre. Les pages du Moniteur résonnent de ce discours qui cherche à exacerber les relations entre les Français et leurs adversaires en guerre. Ce qui est notable, c'est la concentration de cette diatribe sur le caractère de l'adversaire, le refus absolu de dénigrer ses qualités militaires. La Russie, par exemple, est attaquée pour la cruauté de ses soldats, cruauté exemplifiée par la récente campagne russe contre la Pologne et façon sans doute efficace de stimuler la rancœur du soldat français avant même son entrée en guerre ${ }^{21}$. Pour noircir les Autrichiens on peint un tableau funeste de la mentalité anti-française qu'on rencontre à Vienne. 
«La haine contre la France et tout ce qui porte le nom de Français [rapporte-t-on en mars 1793] est tellement à la mode que dans les cercles de la capitale où tout est affaire de ton, comme autrefois en France, on est convenu de proscrire jusqu'aux usages les plus minutieux empruntés de cette nation. On met à l'amende tous ceux qui profèrent un mot de français : les mots mêmes de Monsieur et Madame, reçus en allemand, sont proscrits $»^{22}$.

Avec l'entrée en guerre des Espagnols, on note que cette nation avait doublement trahi la France au moment de l'insurrection à Saint-Domingue, quand ils avaient aidé les « nègres » à « massacrer » les Français au Fort Dauphin $^{23}$. Le but est clair : semer le doute dans l'esprit des soldats sur la fiabilité de l'autre, sur son caractère et ses motifs et encourager l'armée à traiter l'adversaire sans compassion, à s'attaquer à lui sans réserve. En temps de révolution et face à une guerre supposée idéologique, on risque d'aller plus loin, en noircissant tout un peuple comme contre-révolutionnaire ou immoral. C'est un aspect de la propagande révolutionnaire qu'on voit d'abord en Vendée, où le discours officiel cherche à dépeindre le rebelle d'une façon qui le prive de son caractère d'homme et le réduit au rang des bêtes sauvages. On le condamne à faire partie des barbares, comparables aux loups et aux hyènes qui « rôdent » et « sautent sur leur proie », avant de la traîner dans leurs «tanières ». Ces mots ne sont pas innocents; au contraire, ils ont des implications immédiates et très pratiques : on n'est pas obligé de donner aux bêtes la même considération qui est due à un autre être humain. Le langage qui est tenu n'indique pas seulement la hauteur du dédain du bon républicain pour son adversaire, jugé en ce cas comme un insoumis fanatisé, dupe des nobles, des royalistes comme des prêtres insermentés. Il l'autorise à le traiter plus brutalement, à le priver de ses droits de l'homme, bref à perdre ses inhibitions coutumières. Comme s'il ne suffisait pas que le Vendéen soit perçu comme un ennemi idéologique et un traître à la patrie, le discours des décrets, des ordres militaires et des proclamations des députés en mission le prive de son humanité ${ }^{24}$.

Le cas de la Vendée est exceptionnel dans le sens où il s'agit d'une guerre civile, avec toutes les haines qu'une telle guerre déchaîne. Envers les autres pays européens on reste plus modéré, même si dans les descriptions

(22) Moniteur, vol. 15, p. 601.

(23) Ibid., vol. 22, p. 517.

(24) Pour une réflexion sur les individus classés comme « brigands », voir Michael BROERS, Napoleon's Other War : Bandits, Rebels and their Pursuers in the Age of Revolution, Oxford, Oxford University Press, 2010, p. 1-18. 
et les attributions on trouve déjà des échos de la propagande encore plus avilissante qui s'exprimera pendant la Grande Guerre, l'adversaire allemand en Belgique étant décrit comme un massacreur de bébés et un violeur en série, bref comme un monstre d'immoralité qu'on serait en droit d'exterminer. Prenons le cas peut-être le plus extrême, celui de la Grande-Bretagne, victime sans doute d'une anglophobie séculaire ranimée et exagérée pour raisons de guerre. On en décerne des traces dès la déclaration de guerre contre l'Angleterre au printemps de 1793, mais elle réapparaît avec plus d'acharnement quand Londres est soupçonné de financer les guerres des autres pays. Pour les républicains l'image de marque des Anglais est plutôt problématique, car la tradition anglophobe au XVIII ${ }^{\mathrm{e}}$ est contrée par une autre, favorable et admirative : l'anglomanie. Ce dualisme se révèle dans un article du Moniteur consacré à l'insurrection à Toulon en septembre 1793, qui informe ses lecteurs que les nouvelles les plus récentes « feront connaître quels sont ces Anglais qui osaient se décorer du nom de philanthropes » : en effet, une partie importante de l'opinion française associe alors les Anglais à la philanthropie. L'article explique à ses lecteurs la conduite cruelle et barbare des Anglais dans le port méditerranéen.

«Il existe dans la ville, assure-t-on, un tribunal militaire composé d'Anglais et d'Espagnols. Ce tribunal précipite les patriotes dans les cachots, ensuite les embarque on ne sait pour quelle destination ; on évalue le nombre de victimes à huit cent. Pierre Bayle, représentant du peuple, a été trouvé étranglé dans sa prison : on ne sait s'il s'est tué ou si ces monstres l'ont sacrifié ».

Preuve ultime de leur barbarie, « la guillotine a été brûlée, et les perfides qui ont livré Toulon ont le plaisir de voir pendre et rompre leurs concitoyens comme des vilains $»^{25}$.

La principale accusation des républicains, c'est que l'Angleterre avait popularisé la guerre, l'avait rendue nationale, avec la conséquence qu'elle n'était plus seulement l'affaire de Pitt ou des élites londoniennes, mais celle de tout le peuple. Cette conviction prenait ses racines dans la notion très française de la souveraineté du peuple. Or, si à l'époque, quelques-uns croient toujours qu'il faut distinguer la population générale et son gouvernement, il n'y a aucun consensus là-dessus. Les uns acceptent l'idée que le peuple est «trompé par des proclamations mensongères et 
les terreurs hypocrites de son gouvernement », tout en s'assurant que « la nation anglaise, une fois éclairée par notre exemple, fera justice de ses conspirateurs en place $»^{26}$. Mais pour d'autres, cette distinction est sans importance. Le fait même que l'Angleterre jouisse d'institutions constitutionnelles permet aux anglophobes de contester toute distinction entre peuple et gouvernement britanniques. C'est Bertrand Barère qui exprime le plus directement cette idée. "Pourra-t-on encore faire illusion, demande-t-il, avec la distinction usée des peuples et des gouvernements ?» En Angleterre, où le gouvernement se dit national, où le peuple, comme l'exprime si bien Barère, «a une représentation quelconque », comment soutenir que le gouvernement n'est pas représentatif du peuple entier?

«On veut sans cesse séparer le gouvernement de la nation. Eh bien, que le peuple anglais se sépare lui-même de son atroce gouvernement ! Qu'il prouve que son sang et ses trésors ne doivent pas être prodigués dans une guerre contre le droit des hommes et la liberté des nations $»^{27}$.

Par cette logique ce n'est plus seulement la personne de William Pitt, mais le peuple anglais tout entier, qui est visé et qui devient « l'ennemi du genre humain $»^{28}$. Un ennemi, d'ailleurs, qui a des agents partout en Europe, semant la désertion, désorganisant les armées, détruisant l'économie par sa puissance bancaire, sabotant la marine, diffusant la propagande par des pamphlets ou par la presse, jouant les patriotes dans les sociétés populaires ${ }^{29}$.

Si les révolutionnaires stigmatisent des ennemis particuliers en les désignant comme « conspirateurs » ou « barbares », leurs adversaires deviennent aussi des ennemis-types. Les révolutionnaires donnent ainsi une image de l'autre qui leur permet de construire une contre-identité pour la France même. Le plus fréquemment, les ennemis sont dénoncés comme des brigands, des bandits, des hors-la-loi, des hommes organisés en bandes et engagés dans des activités criminelles qui privent de toute légitimité leurs revendications politiques ${ }^{30}$. Le banditisme du XVIII ${ }^{\mathrm{e}}$ est un phénomène bien documenté, surtout dans ces vallées montagneuses et communautés

(26) Sophie WAHNICH, L'impossible citoyen : L'étranger dans le discours de la Révolution française (Paris, 1997), p. 298.

(27) Ibid., p. 306.

(28) Ibid., p. 281.

(29) Norman HAMPSON, The Perfidy of Albion: French Perceptions of England during the French Revolution, Basingstoke, 1998, p. 118-19.

(30) Alan FORREST, «The ubiquitous brigand: the politics and language of repression », dans Charles J. EsDAILE (dir.), Popular Resistance in the French Wars: Patriots, Partisans and Land Pirates, Basingstoke, 2005, p. 25-43. 
autonomes qui caractérisent le sud méditerranéen et les régions frontalières des Alpes et des Pyrénées. Les bandits et les rebelles constitueraient, pour la Révolution et surtout pour les armées de Napoléon, un adversaire souvent formidable, à travers leur histoire à la fois joyeuse et ténébreuse ${ }^{31}$. Mais les comparaisons ne sont pas rigoureuses : dans le discours des républicains, le brigandage est omniprésent, on peut le trouver dans n'importe quelle insurrection ou acte de résistance au régime, et surtout dans les combats de guérilla et de la " petite guerre ». Le brigand ne jouit pas des privilèges accordés au soldat par les règles de la guerre, et sa façon de faire la guerre, se mêlant librement avec la population civile dont il fait partie, permet à l'armée française de l'identifier avec un manque de civilisation, bref avec la barbarie. Cette manière de parler de l'autre est aussi une manière de s'identifier avec les valeurs de la civilisation, et donc de se fournir une légitimation dans les faits. Comme nous le rappelle Michael Jeismann dans son étude sur la notion de l'ennemi national,

« ces attributions linguistiques opposées caractérisent également la figure centrale de l'ennemi qui, depuis la Révolution jusqu'à la fin de la Première Guerre mondiale, joua un rôle crucial dans l'élaboration de l'identité française et représente pour ainsi dire la clé de la sémantique française des oppositions nationales : les barbares $»^{32}$.

Mais que dire des attitudes des soldats eux-mêmes ? Adoptent-ils les attitudes idéologiques de leurs hommes politiques et ne voient-ils en leurs adversaires que des hommes incultes et barbares ? En Vendée, il est vrai, les témoignages ont tendance à prendre à la lettre les dénonciations politiques et à partager les opinions les plus méprisantes quand ils parlent du mode d'engagement des contre-révolutionnaires. « Les brigands ne sont pas les hommes à faire la guerre actuellement comme nous », explique un fusilier dans une lettre à sa sœur ;

« que font-ils, ils se rassemblent au nombre de dix ou douze et suivent une grande route à l'abri de quelques arbres. Voient-ils trois ou quatre pauvres volontaires qui ne sont point armés allant ou venant à l'hôpital [...] ils se montrent et tâchent de les tuer pour avoir ce qu'ils ont, voilà leur beau chef-d'œuvre $»^{33}$.

(31) Michael Broers, Napoleon's Other War, p. 18.

(32) Michael JeISMAnN, La patrie de l'ennemi: la notion d'ennemi national et la représentation de la nation en Allemagne et en France de 1792 à 1918, Paris, CNRS, 1997, p. 127.

(33) Alan ForREST, Napoleon's Men. The Soldiers of the Revolution and Empire, Londres, Hambledon and London, 2002, p. 128. 
Aux yeux des républicains, les Vendéens ne sont que des rebelles, des paysans en armes, des tueurs à la solde des nobles et des prêtres. Ailleurs, on a davantage tendance à oublier la politique et juger l'adversaire d'après des critères plus strictement militaires. Car en Europe on partage une culture militaire qui est largement commune, et qui est pour beaucoup d'officiers la culture du christianisme lui-même. C'est lorsque l'on met le pied au-delà du vieux continent - dans les Caraïbes, par exemple, ou en Russie, parmi des nations asiatiques - qu'on s'approche de l'ennemi avec une peur exagérée. Ces gens, suppose-t-on, ne se battent pas avec les mêmes armes, ne partagent pas le même sens de l'honneur et ne respectent pas les mêmes règles de la guerre.

Un officier français est ainsi outré quand des soldats bashkirs, qui comptent parmi les combattants les plus féroces de la troupe russe, tirent des flèches vers ses hommes ${ }^{34}$. C'est le manque de certitude qui sème l'alarme : on ne sait plus à quoi s'attendre. Mais même la peur ne peut pas cacher une certaine admiration pour la valeur militaire de ces soldats, y compris pour des cosaques que leur réputation de sadisme et de cruauté avait précédés. Les Français reconnaissent dans leur mouvement rapide et leur maîtrise de la tactique du choc des qualités qu'ils ne peuvent que rarement égaler; et ce sont des cavaliers superbes, montés sur les petits chevaux robustes et solides de la steppe. Napoléon lui-même semble reconnaître dans le soldat cosaque un adversaire courageux et un ennemi formidable ${ }^{35}$.

Si les soldats de l'Empire respectent des qualités militaires chez l'adversaire, ils sont souvent plus durs dans leur condamnation de la conduite de la population civile, au moins quand elle prend les armes et cache ou fournit les combattants. D'après leurs codes de conduite militaire, et en accord avec toutes les règles de la guerre internationalement reconnues, il faut maintenir la distinction entre militaires et civils, entre ceux qui font la guerre et ceux auxquels on doit un degré de protection. $\mathrm{Ne}$ pas la respecter, c'est aller vers l'aliénation et déjà franchir la ligne entre la civilisation et la barbarie; c'est aussi exposer le civil à la vengeance de l'armée. Le soldat, soyons clairs, est à tout moment conscient de la différence, qui est à la base de ses impressions à propos d'autres pays et d'autres civilisations. Octave Levavasseur, officier d'artillerie et aide

(34) Heinrich VON Roos, Souvenirs d'un médecin de la Grande Armée, dans Gaspard DUCQUE, Journal de marche du sous-lieutenant Ducque, présenté par Laurent Nagy, Paris, La Vouivre, 2004, p. 92.

(35) Jean Hanoteau (dir.), Memoirs of General de Caulaincourt, Duke of Vicenza, 1812-1813, [1833], London, Cassell, 1935, p. 187. 
de camp du maréchal Ney, est bien placé pour observer le caractère des différents peuples, qu'il commente d'une manière parfois mordante. Dans bien des pays, il se sent rassuré, car « la civilisation des habitants m'avait paru différer peu de la civilisation française » - test toujours essentiel. « L'Allemagne, touchant à notre frontière, a dû prendre quelque chose de nos habitudes, et réciproquement. Les mœurs et les manières des peuples gagnent de proche en proche de Paris jusqu'à Varsovie ; seulement elles se modifient par le climat, par la religion, par les lois $»^{36}$. Au-delà de la Pologne c'est la différence qui frappe le plus.

Mais le sauvage et le barbare n'impliquent pas nécessairement un pays aux marges de l'Europe ou privé de l'influence civilisatrice de Paris. Les grandes villes sont toutes représentées comme civilisées, façonnées par la main des hommes. C'est une des raisons de la stupeur qu'éprouvent les Français quand ils voient, à Moscou, les Russes brûler leur propre capitale. Et les campagnes, même proches de la France, peuvent abriter des coutumes et des superstitions barbares, ou rester sauvages et incultes, emblématiques de l'abandon et de la barbarie. En Espagne, note Levavasseur, « rien n'est semblable à ce qui se remarque ailleurs. À quatre lieues de Bayonne on se croirait à mille lieues de la France », par l'apparence même du paysage.

«Peu de plaines, mais un territoire onduleux et toujours couronné à l'horizon par de hautes montagnes ; des ruisseaux aux nombreuses cascades, mais point d'usines pour les utiliser ; au sommet, des rochers, des maisons ou plutôt des nids d'aigles, construits du temps des Maures, mais, sur les flancs des coteaux, plus de ces villages ni de ces habitations éparses qui donnent au paysage l'animation et la vie ; une terre toujours prête à produire, mais jamais cultivée ; les moutons et les taureaux sauvages en foulent seuls la bruyère éternelle ; la puissance de la nature partout, la force de l'homme nulle part, excepté aux environs des villes, car la main nonchalante de l'Espagnol ne s'étend pas plus loin $»^{37}$.

Cet aspect inculte, sinon désertique, de l'Espagne laisse une forte impression, celle d'un pays qui n'a pas été domestiqué par l'homme et qui reste abandonné à la barbarie. Il contribue à la notion d'une Europe divisée entre un nord avancé et un sud arriéré, une géographie imaginée qui va de

(36) Octave LEVAVASSEUR, Souvenirs militaires d'Octave Levavasseur, officier d'artillerie, aide de camp du maréchal Ney, Paris, Plon-Nourrit, 1914, p. 121-22.

(37) Ibid., p. 122. 
soi avec le commerce et l'industrialisation, bref avec une certaine idée de la modernité.

Cette notion n'est en rien unique, car le monde du XVIII ${ }^{\mathrm{e}}$, soutenu par les idées des Lumières, est attiré par les notions de civilisation et de culture, idées qui se prêtent à une division du continent en régions avancées et arriérées, que ce soit sur le plan économique, religieux ou culturel. L'idée n'est pas sans précédent : au temps de la Renaissance, on avait affirmé que les pays méditerranéens étaient le berceau de la civilisation et de l'art. Mais c'est le XVIII ${ }^{\mathrm{e}}$, surtout l'Allemagne de l'Aufklärung, qui cherche à s'approprier l'idée de civilisation et à l'identifier plus étroitement avec les pays de l'Occident. L'Europe de l'est se trouve bannie de cette Europe de la culture pour être construite dans l'imaginaire de l'ouest comme un pays imparfaitement développé, un pays à mi-voie entre l'Europe du christianisme et l'empire turc, à la fois exotique et barbare. ${ }^{38}$. Le Prussien ou l'Autrichien qui y met le pied se trouve dépaysé, dans un monde largement rural, boueux, misérable, et à ses yeux très sale, dépourvu de maisons solides ou de routes bien construites, bref de toute cette implantation urbaine qu'il associe avec la civilisation. Pour le radical Georg Forster, par exemple, la Pologne se présentait comme un territoire sale et inculte, son peuple « mi-sauvage, mi-civilisé », le pays crasseux « dans le sens moral aussi que physique $»^{39}$. Mais ce n'est pas que l'est qui est associé avec la pauvreté et un manque de culture. Pour l'Européen du nord, accoutumé à une culture plus urbanisée, à des moeurs plus délicates, c'est la campagne, peu dense et inculte, qui sent la barbarie - et cela partout où il passe, semble-t-il, une fois éloigné de chez lui, autant dans les pays méditerranéens qu'à l'est de l'Elbe. Le voyage peut civiliser et faire apprécier la civilisation des autres. Mais il se fait rarement sans des prédispositions et des préjugés à propos des cultures des autres. Et très souvent le voyage du militaire ne fait que confirmer des prédispositions nées dans des bribes de conversation, dans

(38) Larry WOLFF, Inventing Eastern Europe: The Map of Civilisation on the Mind of the Enlightenment, Palo Alto, Stanford University Press, 1994, p. 4.

(39) Vejas Gabriel LiUlevicius, The German Myth of the East, 1800 to the Present, Oxford, Oxford University Press, 2009, p. 51. 
des lectures de jeunesse, ou sur une image d'Épinal offerte un jour de marché par un vendeur itinérant lors de son passage au village.

Alan FORREST

University of York

Centre for Eighteenth Century Studies

The King's Manor, Exhibition Square, York YO1 7EP

alan.forrest@york.ac.uk 